\title{
Copolymerization of n-Butyl Acrylate with Methyl Methacrylate and PMMA Macromonomers: Comparison of Reactivity Ratios in Conventional and Atom Transfer Radical Copolymerization
}

\author{
Sebastian G. Roos and Axel H. E. Muller*,† \\ Institut für Physikalische Chemie, Universität Mainz, Welderweg 15, D-55099 Mainz, Germany \\ Krzysztof Matyjaszewski
}

Department of Chemistry, Carnegie-Mellon University, 4400 Fifth Avenue, Pittsburgh, Pennsylvania 15213

Received December 16, 1998; Revised Manuscript Received August 23, 1999

\begin{abstract}
The reactivity ratios of n-butyl acrylate (nBuA) with methyl methacrylate (MMA) and $\omega$-methacryloyl-PMMA macromonomers (MM) in conventional and atom transfer radical copolymerization (ATRP) have been determined. For the copolymerization of nBuA with MMA, good agreement of the ratios is observed between conventional and controlled radical copolymerization, indicating that chemoselectivities in both processes are similar. The relative reactivity of the $M M\left(1 / r_{n B u A}\right)$ in conventional copolymerization is significantly lower than that of MMA. It depends on the concentration of the comonomers but is not significantly influenced by the length of the MM. At high concentrations the relative reactivity decreases due to diffusion control of the MM addition. In ATRP the relative reactivity of the $\mathrm{MM}$ is much nearer to the value of MMA. This is explained by the different time scales of monomer addition in both processes: whereas the frequency for monomer addition is in the range of milliseconds for conventional polymerizations, it is in the range of seconds in ATRP; thus, diffusion control is less important here. This gives the opportunity to copolymerize at much higher concentrations than in conventional radical copolymerization. In addition, the graft copolymers obtained by ATRP have lower polydispersities.
\end{abstract}

\section{Introduction}

Graft copolymers offer all properties of block copolymers but are usually easier to synthesize. Moreover, the branched structure leads to decreased melt viscosities which is an important advantage for processing. Depending on the nature of their backbone and side chains, they can be used for a wide variety of applications, such as impact-resistant plastics, thermoplastic elastomers, compatibilizers, and polymeric emulsifiers. According to the theory of Erukhimovich, ${ }^{1}$ graft copolymers should show better phase separation than triblock copolymers.

The state-of-the-art technique to synthesize graft copolymers is the copolymerization of macromonomers (MM) with low molecular weight monomers. ${ }^{2}$ It allows for the control of the polymer structure which is given by three parameters: (i) chain length of side chains, which can be controlled by the synthesis of the macromonomer by living polymerization; (ii) chain length of backbone, which can be controlled in a living copolymerization; (iii) average spacing of the side chains, which is determined by the molar ratio of the comonomers and the reactivity ratio of the low-molecularweight monomer, $r_{1}=k_{11} / k_{12}$. However, the distribution of spacings may not be very easy to control due to the incompatibility of the polymer backbone and the macromonomers.

In the past, we have used conventional radical copolymerization for the synthesis of graft copolymers. ${ }^{3,4}$ Obviously, a control of backbone chain length is not

\footnotetext{
† New address: Macromolecular Chemistry II, University of Bayreuth, D-95440 Bayreuth. *Corresponding author. e-mail axel.mueller@uni-bayreuth.de.
}

possible for this mechanism. Various strategies have been developed recently for a controlled radical polymerization. ${ }^{5}$ One approach is atom transfer radical polymerization (ATRP) ${ }^{6,7}$ Dormant halogenated initiator or chain ends are reversibly activated by a transitionmetal complex, e.g., $\mathrm{CuX} \cdot \mathrm{L}_{n}(\mathrm{X}=\mathrm{Cl}$ or $\mathrm{Br}, \mathrm{L}=$ ligand, $\mathrm{n}=1$ or 2), via halogen atom transfer. Bimolecular terminations are minimized due to the low concentration of free radicals. A homogeneous ATRP of MMA is reached by using $\alpha$-halogenated esters ${ }^{8}$ or $p$-toluenesulfonyl chl oride ${ }^{9}$ as initiators and 4,4'-di (5-nonyl)-2,2' bipyridine (dN bipy) as ligand resulting in polymers of low polydispersity $\left(\overline{\mathrm{M}}_{\mathrm{w}} / \overline{\mathrm{M}}_{\mathrm{n}}<1.05\right)$ and molecular weights up to $M_{n}=10^{5}$.

To obtain a better control of backbone chain length, we have used ATRP to synthesize graft copolymers. It should be noted that conventional and controlled polymerizations lead to different copolymer structures. In conventional radical copolymerization the polymers show a chemical heterogeneity of first order. The chemical composition of different polymer molecules is different due to the short period of time needed to form a polymer and the shift of the comonomer ratio during polymerization. In a living polymerization all chains grow simultaneously with the same chemical composition, but this changes during the polymerization leading to a heterogeneity of second order, i.e., a compositional shift within all of the chains.

To control the structure of graft copolymers, the reactivity ratios of the copolymerization of $\mathrm{nBuA}$ and PMMA-MM in the ATRP were investigated and compared to those obtained in conventional radical copolymerization. For comparison, the same was done for the copolymerization of $\mathrm{nBuA}$ and MMA. 


\section{Experimental Section}

Reagents. n-Butyl acrylate (nBuA, BASF AG) was fractionated from $\mathrm{CaH}_{2}$ over a $1 \mathrm{~m}$ column filled with Sulzer packing at $45 \mathrm{mbar}$, stirred over $\mathrm{CaH}_{2}$, degassed, and distilled in high vacuum. $\mathrm{CuBr}$ (95\%, Aldrich) was purified by stirring overnight in acetic acid. After filtration it was washed with ethanol, ether, and then dried.

4,4'-Di(5-nonyl)-2,2'-bipyridine (dN bipy) was prepared by coupling of 4-(5-nonyl)pyridine with a 5\% Pd/C catalyst at 200 ${ }^{\circ} \mathrm{C}$ for 7 days. ${ }^{10}$ Purification was achieved by distillation of the product at $10^{-2}$ mbar. Ethyl acetate (Aldrich), copper powder (99\% for organic synthesis, Aldrich), and methyl 2-bromopropionate (MBP, Aldrich) were used as received. Methacryloylterminated PMMA macromonomers of low polydispersity were prepared by group transfer polymerization using a trimethylsiloxy-functionalized initiator. After polymerization the trimethoxy protecting group was removed, and the resulting $\mathrm{OH}$ terminated PMMA was reacted with methacryloyl chloride. ${ }^{11}$ About ca. every sixth macromonomer $\left(\mathrm{MM}, \overline{\mathrm{M}}_{\mathrm{n}}=10900\right.$, $\left.\bar{M}_{w} / \bar{M}_{n}=1.19\right)$ was labeled with naphthylcarboxyethyl methacrylate in order to facilitate the determination of the MM conversion by using a UV detector.

Copolymerizations. All solid chemicals were weighed into an ampule and degassed. In a glovebox all degassed liquids were added to the ampule, which was sealed and placed in an oil bath held at the desired temperature. Conventional radical copolymerizations were performed in butyl acetate at $60{ }^{\circ} \mathrm{C}$ using AIBN as initiator. ATRP was performed in ethyl acetate at $90^{\circ} \mathrm{C}$ using MBP as initiator, $\mathrm{CuBr}$ as catalyst, and $\mathrm{dN}$ bipy as ligand. For ATRP all kinetic samples were taken from the same ampule by cooling to room temperature in the glovebox, withdrawing an aliquot, and placing the ampule afterward into the oil bath again. For conventional radical polymerization several ampules were placed in the oil bath and were taken out after certain times. The ATRP reaction solution was diluted with THF and filtered over a short alumina column. After evaporation of the solvent, all polymers were dissolved in benzene, filtered, and freeze-dried.

Analysis. Comonomer conversions were determined from the reaction solution by GC using a methylpolysiloxane capillary column. Decane was used as an internal standard. The conversion of the macromonomer was determined by GPC analysis of the copolymer using UV $(\lambda=280 \mathrm{~nm})$ and RI detectors. GPC was performed using THF as eluent at a flow rate of $1 \mathrm{~mL} / \mathrm{min}$. Detectors: $2 \times$ J asco-U videc 100 III with variable wavelength, Bischoff RI detector 8110 , and column set: $2 \times 60 \mathrm{~cm}, 5 \mu$ PSS SDV gel, $100 \AA$ and linear: $10^{2}-10^{5}$ $\AA$. PMMA and PnBuA standards made by anionic polymerization $^{12}$ were used for calibration. For graft copolymers the calibration curve for linear PnBuA were used. Thus, the molecular weight averages are only apparent ones. The $\bar{M}_{n}$ and the PDI of the pure copolymer were determined by subtracting the signal of residual MM from that of the crude copolymer.

\section{Results and Discussion}

Determination of Reactivity Ratios of MMA and nBuA in ATRP. The monomer reactivity ratios were obtained by two different methods by using methyl 2-bromopropionate (MBP) as initiator and CuBr/dNbipy as catalyst: the J aacks method ${ }^{13}$ for high monomer feed ratios ([nBuA $]_{0} /[\mathrm{MMA}]_{0}=8$ and 0.1 , respectively) and the Kelen-Tüdös ${ }^{14}$ method with varying feed monomer composition. For the Kelen-Tüdös method three kinetic experiments were performed. After each withdrawal the residual comonomer concentration was determined and regarded as the monomer feed for the next data point. The data were also treated using nonlinear optimization. ${ }^{19}$ No differences were found to the Kelen-Tüdös values.The comparison of the two different methods

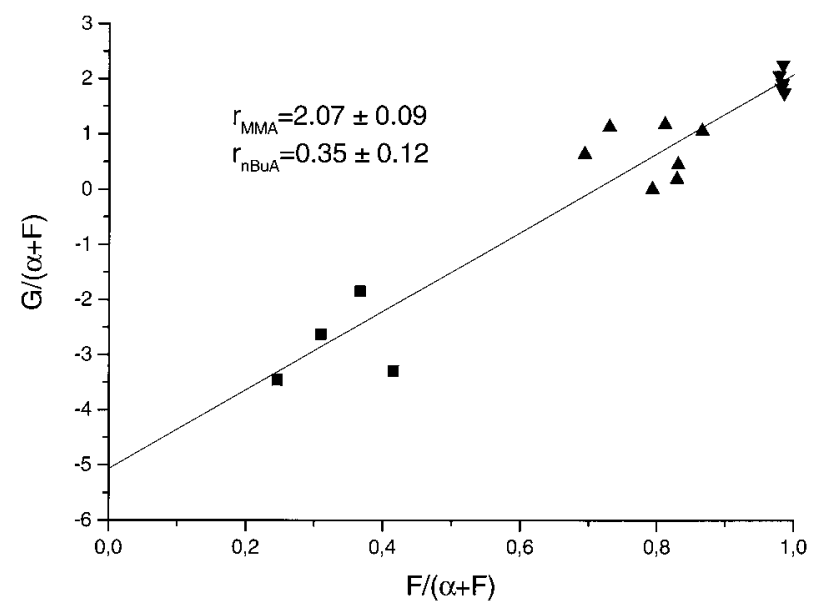

Figure 1. Kelen-Tüdös plot for the atom transfer radical copolymerization of $\mathrm{nBuA}$ and MMA in ethyl acetate at $90^{\circ} \mathrm{C}$ $(\alpha=1)$.

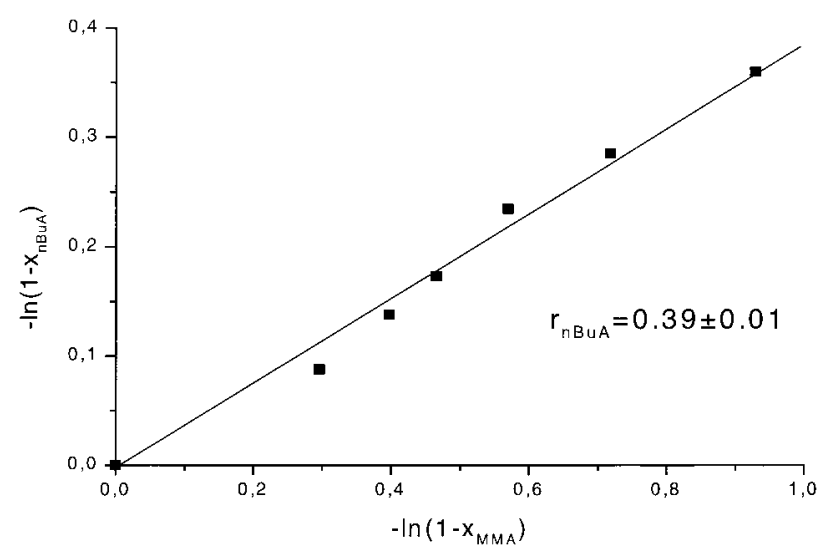

Figure 2. J aacks plots of the ATRP at monomer ratio $[\mathrm{nBuA}]_{0}:[\mathrm{MMA}]_{0}=8$.

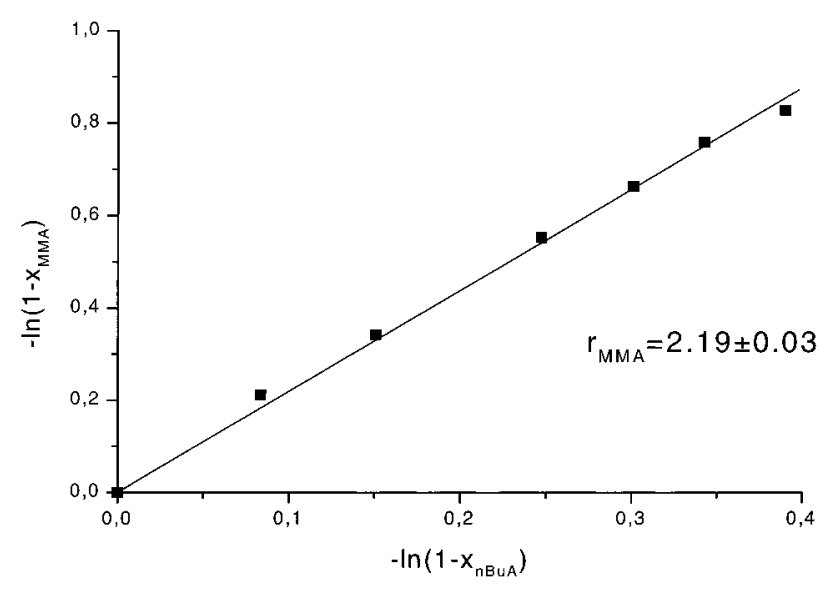

Figure 3. J aacks plots of the ATRP at monomer ratio $[\mathrm{nBuA}]_{0}:[\mathrm{MMA}]_{0}=0.1$.

shows good agreement of the reactivity ratios obtained by both methods. In Table 1 these parameters are compared to an average of the known literature data of conventional radical copolymerization of the mentioned monomers determined by the Kelen-Tüdös method and nonlinear optimization. The nonlinear optimization produces more accurate values.

It has to be mentioned that the reactivity ratios of the conventional polymerizations were obtained at different temperatures and solvents. However, the ratios should not depend significantly on these parameters within the limits of experimental error. The ratios show 
Table 1. Reactivity Ratios of the Copolymerization of the Conventional and Controlled Radical Polymerization of nBuA and MMA

\begin{tabular}{llllll}
\hline & \multicolumn{2}{c}{ ATRP } & & \multicolumn{2}{c}{$\begin{array}{c}\text { conventional radical } \\
\text { polymerization }\end{array}$} \\
\cline { 2 - 3 } \cline { 5 - 6 } $\begin{array}{l}\text { system } \\
\text { method }\end{array}$ & Kelen-Tüdös & J aacks & & Kelen-Tüdösa & $\begin{array}{c}\text { nonlinear } \\
\text { optimization }\end{array}$ \\
\hline ref & this paper & this paper & & $15-18$ & 19 \\
r $_{\text {nBuA }}$ & $0.36 \pm 0.12$ & $0.39 \pm 0.01$ & & $0.26 \pm 0.14$ & $0.30 \pm 0.03$ \\
r $_{\text {MMA }}$ & $2.07 \pm 0.09$ & $2.19 \pm 0.03$ & & $2.15 \pm 0.37$ & $1.79 \pm 0.18$
\end{tabular}

a Mean and standard deviation of six values.

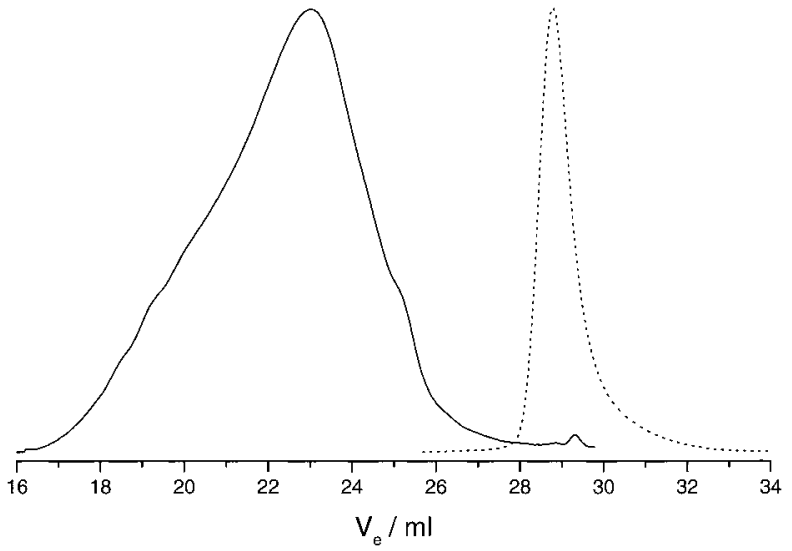

Figure 4. Apparent molecular weight distribution of PnBuAg-PMMA graft copolymer obtained by conventional radical copolymerization (-; $\bar{M}_{n, a p p}=158000, \bar{M}_{w} / \bar{M}_{n}=3.1$ ) and PMMA macromonomer used $\left(\cdots ; \bar{M}_{\mathrm{n}, \mathrm{MM}}=10900, \bar{M}_{\mathrm{w}} / \overline{\mathrm{M}}_{\mathrm{n}}=\right.$ 1.19).

a good agreement for both polymerization techniques, so we conclude that the different mechanisms do not affect the propagation reaction. This means that the equilibrium between dormant and active species does not effect the sel ectivity of the growing radicals. Similar observations have been made with other comonomer pairs. ${ }^{20-24}$ Using $\mathrm{NiBr}_{2}\left(\mathrm{PPh}_{3}\right)_{2}$ as catalyst, Moineau et al ${ }^{31}$ recently reported similar reactivity ratios $\left(r_{\mathrm{MMA}}=\right.$ $\left.1.7 ; r_{\text {nBuA }}=0.34\right)$.

Conventional Radical Copolymerization of $\mathrm{nBuA}$ with PMMA-MM. Figure 4 shows a typical broad molecular weight distribution of a PnBuA-g-PMMA graft copolymer and that of the PMMA-MM used.

Figure 5 shows a typical time-conversion plot. The macromonomer is converted faster than nBuA due to the methacryloyl end group of the MM. Figure 6 shows the accompanying J aacks plot for determination of the relative reactivity, $1 / r_{n B u A}$.

The dependence of the relative reactivity of the PMMA-MM on the total weight concentration of monomers, $w_{n B u A}+w_{M M}$, was studied. The reciprocal value of $r_{\mathrm{nBuA}}$ (as determined by the J aacks method) is equival ent to the relative reactivity of the $M M\left(1 / r_{n B u A}\right.$ $\left.=\mathrm{k}_{\mathrm{nBuA}}-\mathrm{mm} / \mathrm{k}_{\mathrm{nBuA}}-\mathrm{nBuA}\right)$. In all copolymerizations with nBuA the reactivity of the $\mathrm{MM}\left(1 / \mathrm{r}_{\mathrm{nBuA}}<1.6\right)$ is much lower than that of MMA $\left(1 / \mathrm{r}_{\mathrm{nBuA}} \approx 3.3^{19}\right)$.

Figure 7 shows that the reactivity of the $\mathrm{MM}$ initially increases with increasing total concentration of monomers (and thus total concentration of polymer). After reaching a maximum the reactivity decreases at very high concentration of monomers. Let us first discuss the reactivity decrease at high concentration. A similar effect was observed by Radke and $\mathrm{Müller}^{25}$ in the copolymerization of PMMA-MM with MMA. It was attributed to the increased viscosity of the reaction

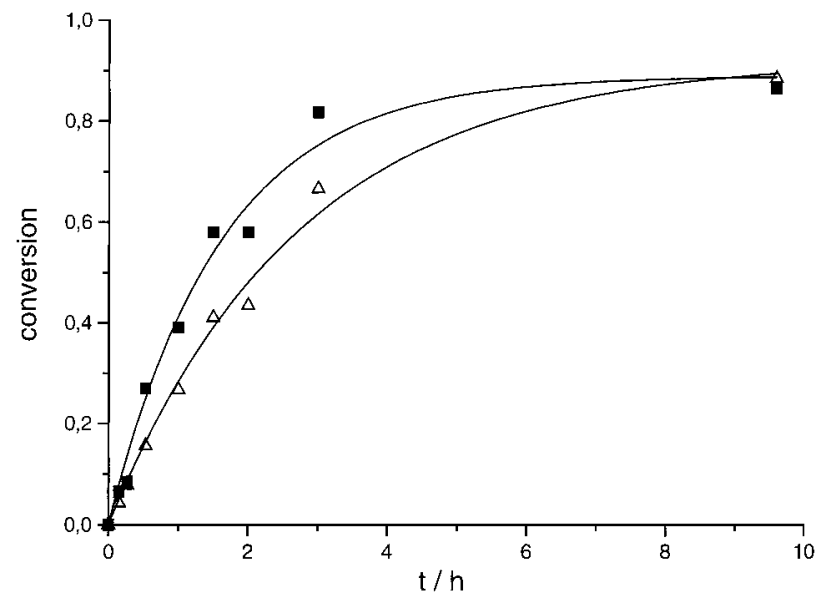

Figure 5. Time-conversion plot of the conventional radical

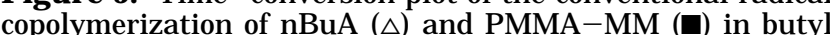
acetate at $60^{\circ} \mathrm{C}$. $[\mathrm{nBuA}]_{0}=0,77 \mathrm{~mol} / \mathrm{L},[\mathrm{nBuA}]_{0} /[\mathrm{MM}]_{0}=170$, $[\mathrm{AIBN}]_{0}=5 \times 10^{-3} \mathrm{~mol} / \mathrm{L} ; \bar{M}_{\mathrm{n}, \mathrm{MM}}=10900$.

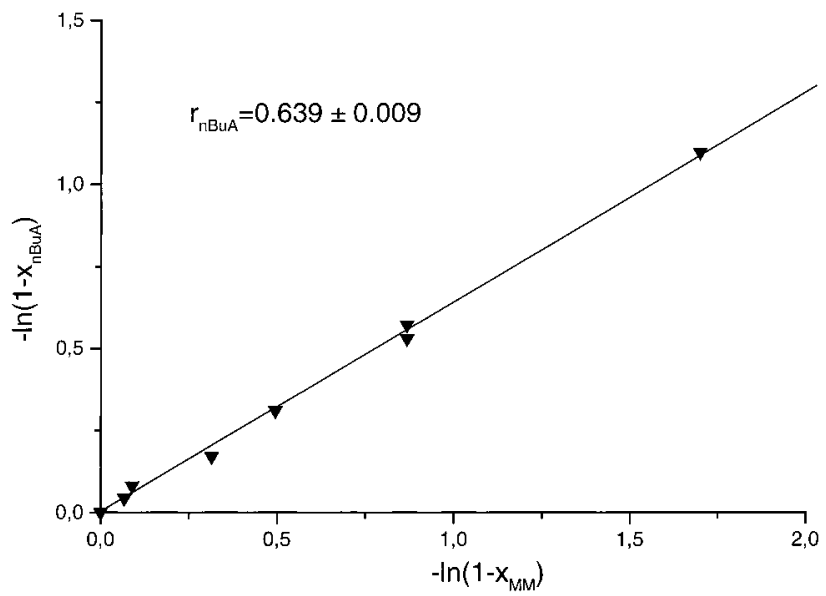

Figure 6. J aacks plot obtained from the time-conversion plot in Figure 5.

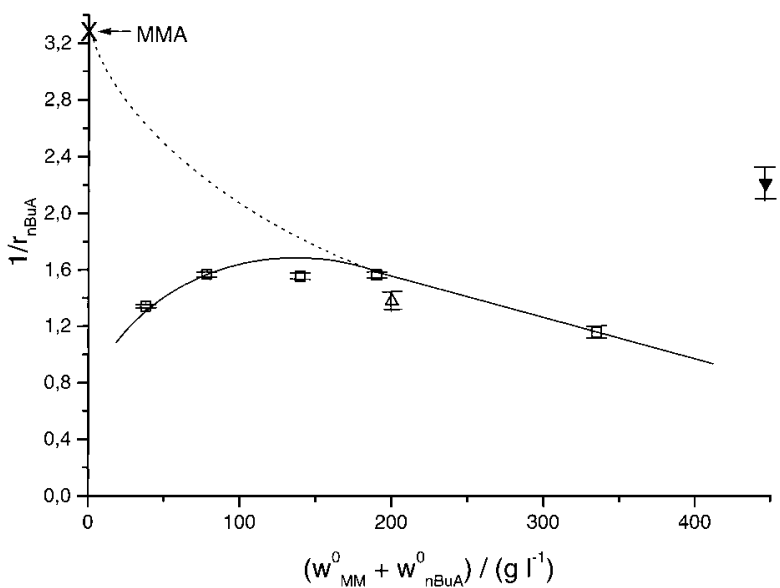

Figure 7. Dependence of PMMA macromonomer reactivity on the total initial concentration of monomers. Conventional radical copolymerization with $\mathrm{nBuA}$ in butyl acetate at $60^{\circ} \mathrm{C}$ with $[\mathrm{nBuA}]_{0} /[\mathrm{MM}]_{0}=170$ and $\bar{M}_{\mathrm{n}, \mathrm{MM}}=10900(\square)$ and $\overline{\mathrm{M}}_{\mathrm{n}, \mathrm{MM}}$ $=5600(\triangle),[\mathrm{AIBN}]_{0}=5 \times 10^{-3} \mathrm{~mol} / \mathrm{L}$. ATRP with nBuA in ethyl acetate at $90{ }^{\circ} \mathrm{C}$ with $[\mathrm{nBuA}]_{0}=2.33 \mathrm{~mol} / \mathrm{L},[\mathrm{MPB}]_{0}=$ $4.66 \times 10^{-3} \mathrm{~mol} / \mathrm{L},[\mathrm{CuBr}]=[\mathrm{Cu}]=4,66 \times 10^{-2} \mathrm{~mol} / \mathrm{L},[\mathrm{nBuA}]_{\mathrm{o}}$ $[\mathrm{MM}]_{0}=83$, and $\bar{M}_{n, M M}=5600(\nabla) ;(\times)$ copolymerization with MMA.

solution leading to diffusion control of the mobility of the MM lowering its apparent reactivity. At very high concentrations ( $3 \mathrm{~mol} / \mathrm{L})$ the resulting product was 


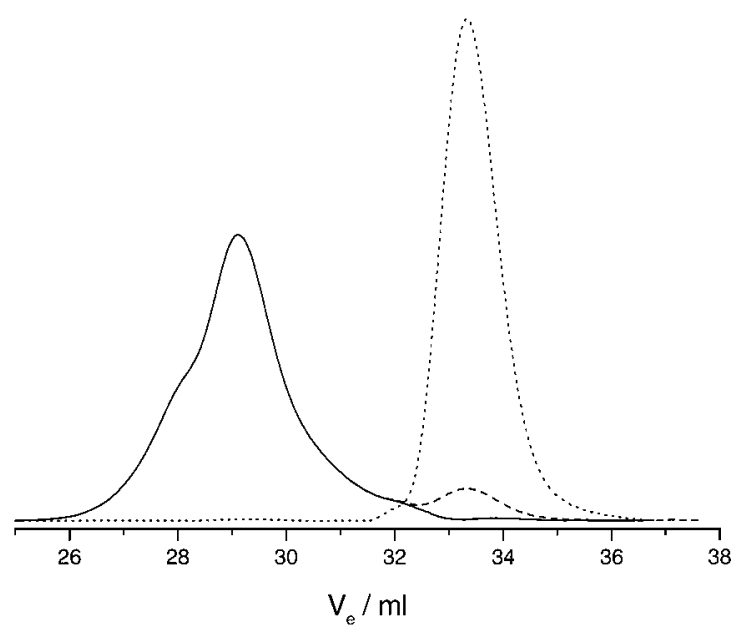

Figure 8. GPC eluograms of the crude $\mathrm{PnBuA}-\mathrm{g}-\mathrm{PMMA}$ graft copolymer (- - ), the pure graft copol ymer $\left(-; \bar{M}_{n, a p p}=47200\right.$, $\left.\bar{M}_{w} / \bar{M}_{n}=1.66\right)$, and macromonomer used $\left(\cdots ; \bar{M}_{n, M M}=5600\right.$, $\left.\bar{M}_{\mathrm{w}} / \bar{M}_{\mathrm{n}}=1.16\right)$.

reported to be a polymer blend of $\mathrm{PnBuA}$ and unreacted PMMA-MM, which was not incorporated into the backbone. ${ }^{3}$ The initial increase of the reactivity is in excellent agreement with earlier results on the conventional copolymerization of $\mathrm{nBuA}$ and PMMA-MM in toluene. ${ }^{26}$ Since the $\mathrm{MM}$ and the resulting graft copolymers are incompatible, the polymer coils will try to avoid contacts at low concentration. Thus, the actual concentration of macromonomers near the growing radical will be lower than its stoichiometric concentration, similar to Harwood's "bootstrap" model. ${ }^{27} \mathrm{H}$ owever, no evidence for micelle formation could be found by viscosity measurements, in contrast to reports on the copolymerization of the $\omega$-styryl macromonomer of poly(2,6-dimethyl1,4-phenylene ether) and MMA. ${ }^{28}$ With increasing polymer concentration the polymer coils start to overlap, and the "bootstrap effect" will become less important than the viscosity effect. Using two macromonomers of different length, it was shown that $1 / r$ does not strongly depend on the MM chain length (see Figure 7). Further experiments showed that the molar ratio of comonomers also does not strongly effect the MM reactivity.

Copolymerization by ATRP. nBuA and PMMA$M M$ were copolymerized by ATRP in ethyl acetate at $90{ }^{\circ} \mathrm{C}\left([\mathrm{nBuA}]_{0}:[\mathrm{MM}]_{0}:[\mathrm{MBP}]_{0}:[\mathrm{CuBr}]_{0}:[\mathrm{dN} \text { bipy }]_{0}:[\mathrm{Cu}]_{0}=\right.$ 500:5.8:1:10:20:10). Metallic copper was added in order to reduce $\mathrm{CuBr}_{2}$ formed via termination reactions. ${ }_{2}^{29} \mathrm{The}$ $M M$ used had $\bar{M}_{n}=5600$. F or the calculation of $\overline{\mathrm{M}}_{n}$ and PDI of the pure graft copolymer the unreacted UVlabeled macromonomer was numerically subtracted from the crude copolymer.Figure 9 shows the conversi ons of the comonomers versus time. The macromonomer is first converted faster than nBuA due to its more reactive methacryloyl end group. The conversion of the MM stopped at $90 \%$ due to the very high viscosity of the reaction solution (ca. 45\% solid content) which reduces the mobility of the $\mathrm{MM}$.

Figure 10 shows the apparent molecular weights and polydispersities of the pure copolymers (after subtracting residual $\mathrm{MM}$ ) versus the average conversion $\left(w^{0}{ }_{n B U A} x_{p, n B U A}+w^{0}{ }_{M M} x_{p, M M}\right) /\left(w^{0}{ }_{n B U A}+w^{0}{ }_{M m}\right)$. With increasing conversion the molecular weight raises linearly, indicating a controlled copolymerization. However, the PDI increases slowly with conversion, indicating some side reactions. Both termination and transfer to polymer ${ }^{30}$ could contribute to this effect.

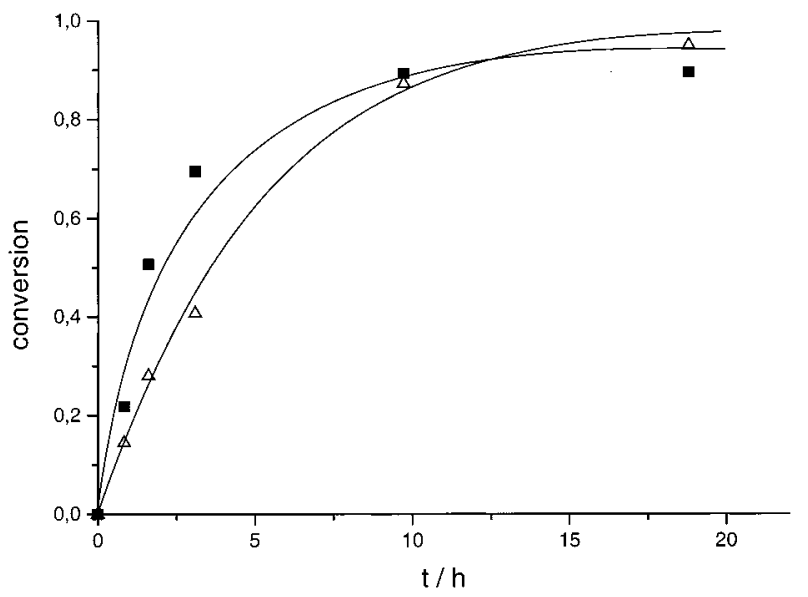

Figure 9. Time-conversion plot of the atom transfer radical copolymerization of nBuA $(\triangle)$ and PMMA-MM ( $\square)$, synthe sized with $[\mathrm{nBuA}]_{0}=2.33 \mathrm{~mol} / \mathrm{L},[\mathrm{nBuA}]_{0} /[\mathrm{MM}]_{0}=83$ in ethyl acetate at $90^{\circ} \mathrm{C}$.

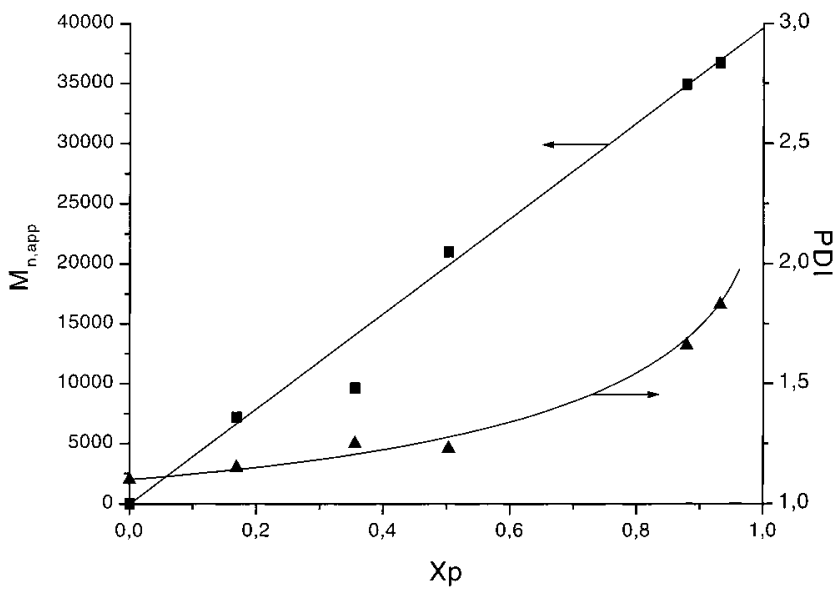

Figure 10. Apparent number-average molecular weight and polydispersity index versus average conversion for the timeconversion plot in Figure 9.

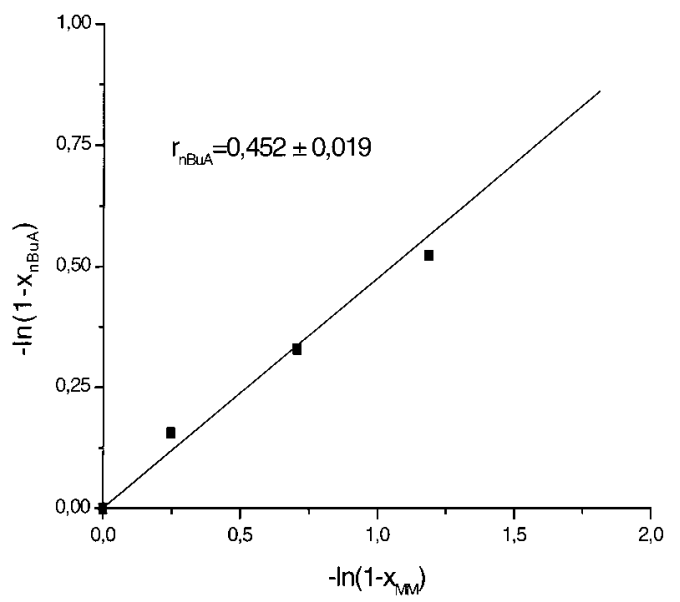

Figure 11. J aacks plot obtained from the time-conversion plot in Figure 9.

Figure 11 shows the J aacks plot obtained from the experiment of Figure 9 . The relative reactivity of the MM $\left(1 / r_{\mathrm{nBuA}}=2.2\right)$ is nearly as high as that of MMA $\left(1 / r_{\mathrm{nBuA}}=2.7\right.$, Table 1$)$. It is significantly higher than that found in conventional radical polymerization at comparable concentrations $\left(1 / \mathrm{r}_{\mathrm{nBuA}} \approx 1.2\right.$, Figure 7$)$.

In conventional radical polymerization the lower reactivity of macromonomers compared to that of MMA 
was explained by the diffusion control of the reaction. One polymer molecule is built in less than a few seconds, and the time interval between two consecutive monomer additions is in the range of milliseconds. Thus, a MM has only a short time to move to the reactive chain end. In ATRP it takes hours to build the same degree of polymerization due to the reversible deactivation. Thus, the time interval for monomer addition is in the range of seconds to minutes, leaving enough time for the monomer and macromonomer to move to the dormant chain end. Once the dormant chain end is converted to a radical, the $\mathrm{MM}$ has the same chance to react as the low molecular weight monomer. Consequently, the relative reactivity is closer to the value for the low molecular weight model, MMA.

A comparison of Figures 4, 8, and 10 reveals that the MWDs of the copolymers obtained in ATRP are significantly narrower (although not extremely narrow) than those obtained in conventional radical copolymerization. Two-dimensional chromatography of the products showed that the polymers obtained in ATRP are much more homogeneous in structure than those obtained by conventional polymerization. ${ }^{32}$

\section{Conclusions}

Both conventional and controlled radical polymerization result in comparable reactivity ratios for low molecular weight monomers where diffusion control is absent. This indicates that the selectivity of the corresponding radicals is independent of the mechanism. The relative reactivity of macromonomers, however, strongly decreases at high concentrations in the conventional radical copolymerization of $\mathrm{nBuA}$ and $\mathrm{PMMA}-\mathrm{MM}$, due to the diffusion control. In controlled radical copolymerization a much higher relative reactivity of the MM is observed at these concentrations. This is explained by different time scales for monomer addition leading to the absence or a much later onset of diffusion control in ATRP. Accordingly, copolymerization of macromonomers is facilitated in ATRP. As a result, lower polydispersities of the polymers are observed.

Acknowledgment. This work was supported by the Deutsche Forschungsgemeinschaft within the Sonderforschungsbereich 262. S. Roos expresses warm thanks to Prof. K. Matyjaszewski for accepting him to his research group at Carnegie-M ellon University for three months.

\section{References and Notes}

(1) Dobrynin, A. V.; Erukhimovich, I. Y. Macromolecules 1993, $26,276$.
(2) Schulz, G. O.; Milkovich, R. J . Appl. Polym. Sci. 1982, 27, 4773.

(3) Roos, S.; Müller, A. H. E.; Kaufmann, M.; Siol, W.; Auschra, C. In Applications of Anionic Polymerization Research; Quirk R. P., Ed.; ACS Symp. Ser. 1998, 696, 208.

(4) Radke, W.; Roos, S.; Stein, H. M.; Müller, A. H. E. Macromol. Symp. 1996, 101, 19.

(5) Matyjaszewski, K. In Controlled Radical Polymerization; ACS Symp. Ser. 1998, 685.

(6) Kato, M.; Kamigaito, M.; Sawamoto, M.; Higashimura, T. Macromolecules 1995, 28, 1721.

(7) Wang, J .-S.; Matyjaszewski, K. J . Am. Chem. Soc. 1995, 117, 5614.

(8) Grimaud, T.; Matyjaszewski, K. Macromolecules 1997, 30, 2216.

(9) Percec, V.; Barboiu, B.; Kim, H.-J . J . Am. Chem. Soc. 1998, 120, 305.

(10) Matyjaszewski, K.; Patten, T. E.; Xia, J . J . Am. Chem. Soc. 1997, 119, 674

(11) Oare, D. A.; Heathcock, C. H. J . Org. Chem. 1990, 55, 157.

(12) Schmitt, B.; Müller, A. H. E., unpublished results.

(13) J aacks, V. Makromol. Chem. 1972, 161, 161.

(14) Kelen, T.; Tüdös, F.; Turcsányi, B.; Kennedy, J . P. J . Polym. Sci., Polym. Chem. Ed. 1977, 15, 3047.

(15) Bevington, J . C.; Harris, D. O. Polym. Lett. 1967, 799.

(16) Brosse, J .-C.; Gauthier, J .-M.; Lenain, J .-C. Macromol. Chem. 1983, 184, 505.

(17) Emelie, B.; Pichot, C.; Guillot, J . Makromol. Chem. 1991, 192, 1629.

(18) Grassie, N.; Torrance, B. J . D.; Fortune, J . D.; Gemmell, J . D. Polymer 1965, 6, 653.

(19) Dube, M. A.; Penlidis, A. Polymer 1995, 36, 587.

(20) Greszta, D.; Matyjaszewski, K. Polym. Prepr. 1996, 37 (1), 569.

(21) Arehart, S. V.; Greszta, D.; Matyjaszewski, K. Polym. Prepr. 1997, 38 (1), 705.

(22) Matyjaszewski, K. Macromolecules 1998, 31, 4710.

(23) Haddleton, D.; Crossman, M. C.; Hunt, K. H.; Topping, C.; Waterson, C.; Suddaby, K. S. Macromolecules 1997, 30, 3992.

(24) Kotani, Y.; Kamigaito, M.; Sawamoto, M. Macromolecules 1998, 31, 5582.

(25) Radke, W.; Müller, A. H. E. Makromol. Chem., Macromol. Symp. 1992, 54/ 55, 583.

(26) Stein, H. M. Diplomarbeit, Universität Mainz, 1992.

(27) Harwood, J . Makromol. Chem., Macromol. Symp. 1987, 10/ 11, 331.

(28) Percec, V.; Wang, J . H. Makromol. Chem., Macromol. Symp. 1992, 54/ 55, 561.

(29) Matyjaszewski, K.; Coca, S.; Gaynor, S. G.; Wei, M.; Woodworth, B. E. Macromolecules 1997, 30, 7348.

(30) Ahmad, N. M.; Heatley, F.; Lovell, P. A. Macromol ecules 1998 31, 2822.

(31) Moineau, G.; Minet, M.; Dubois, P.; Teyssié, P.; Senninger, T.; J érôme, R. Macromol ecules 1999, 32, 27.

(32) Roos, S. G.; Schmitt, B.; Müller, A. H. E. Polym. Prepr. (Am. Chem. Soc., Div. Polym. Chem.) 1999, 40 (2), 140.

MA9819337 